\title{
DSM-III-R and ICD-10 diagnoses of schizophrenia had higher predictive validity than that of ICD-9 and CATEGO S+ diagnoses
}

Mason P, Harrison G, Croudace T, et al. The predictive validity of a diagnosis of schizophrenia. A report from the International Study of Schizophrenia (ISoS) coordinated by the World Health Organisation and the Department of Psychiatry, University of Nottingham. Br J Psychiatry. 1997 Apr $; \mathbf{1 7 0 : 3 2 1 - 7 . ~}$

\section{Objective}

To test the predictive validity of 4 definitions of schizophrenia using 4 diagnostic classifications: Diagnostic and Statistical Manual of Mental Disorders, 3rd edition, revised (DSM-III-R); tenth and ninth revisions of the International Classification of Diseases (ICD-10 and ICD-9); and a restrictive CATEGO S + .

\section{Design}

Inception cohort followed up for 13 years.

\section{Setting}

Community psychiatric service, Nottingham, UK.

\section{Patients}

99 patients (mean age $30 \mathrm{y}, 66 \%$ men) making their first contact for an episode of psychosis between 1 August 1978 and 31 July $1980.96 \%$ completed follow up.

\section{Assessment of prognostic factors}

All patients were interviewed by a psychiatrist using the Present State Examination and the Psychological Impairments Rating Scale. Other schedules covering psychiatric, personal, family, and social background and social disability were completed by a different interviewer with a close relative of the patient or other informant. Each definition of schizophrenia (ICD-9, ICD-10, CATEGO S +, and DSM-III-R) was applied to each patient after the initial assessments. To study the effects of 6 month duration criteria, duration adjusted definitions of schizophrenia were created for each scale.

\section{Main outcome measures}

The ability of the 4 definitions to predict 13 year outcome using the Global Assessment of Functioning scales.

\begin{abstract}
Main results
DSM-III-R and ICD-10 diagnoses of schizophrenia had high predictive validity for symptoms and disability and for 13 year outcome; both provided stable diagnoses. DSM-III-R proved to be considerably less sensitive than $I C D-10$. An $I C D-9$ diagnosis of schizophrenia was less discriminating, especially for symptoms, and a CATEGO S + definition had no predictive validity. The addition of the 6 month duration criteria improved the predictive validity of $I C D-10$ and $I C D-9$ and, to a lesser degree, the predictive validity of CATEGO $\mathrm{S}+$. Removing the duration criteria from $D S M-I I I-R$ reduced the predictive validity, particularly for symptomatic outcome.
\end{abstract}

\section{Conclusions}

Diagnostic and Statistical Manual of Mental Disorders, 3rd edition, revised (DSM-III-R) and tenth revision of the International Classification of Diseases (ICD-10) diagnoses of schizophrenia had high predictive validity and were superior to ICD-9 and CATEGO S + diagnoses. ICD-10 had superior sensitivity to DSM-III-R.

Sources of funding: World Health Organisation; U.S. Laureate Foundation; participating field research centres.

For article reprint:Dr P Mason, Department of Psychiatry, Royal Liverpool University Hospital, Liverpool L69 $3 B X, U K$.

Abstract and commentary also published in Evidence-Based Medicine 1997 Nov-Dec;2:190.

\section{Commentary}

As yet, no clinical or pathological gold standard exists for the diagnosis of schizophrenia, and the validity of diagnostic classification rests to some extent on its ability to predict outcome. Research over 25 years has shown that Schneider's "first-rank" symptoms are not exclusive to schizophrenia and their presence during an acute illness does not predict a stable diagnosis over time. ${ }^{1}$ Classifications have evolved minimum duration criteria and now include negative symptoms, such as flat affect and lack of speech, as well as the positive symptoms of hallucinations and delusions. Studies like this are important in testing the resulting predictive validity. Strengths of the study by Mason et al include a complete and representative inception cohort, a high follow up rate, and good inter-rater reliability for diag- nosis and follow up assessments. Diagnosis was blinded to outcome status. The authors acknowledge a modest sample size and concede that their findings may not be generalisable outside the setting of a single community-based service.

The findings confirm that CATEGO $\mathrm{S}+$, which is based on first-rank symptoms, has poor predictive validity, and that ICD-10 is better than ICD-9. The addition of the DSM-III-R criterion of 6 month duration improves the prediction of poor long term outcome; however, it is more restrictive and seems to miss a substantial proportion of patients in the early phase who prove to have schizophrenia in the longer term.

$D S M-I V$, the latest US classification, specifies virtually the same symptoms as ICD-10 for the same 1 month period but retains the overall requirement for a 6 ,month duration, which can include less specific prodromal symptoms. ${ }^{2}$ This study suggests that ICD-10 should be preferred for studies needing high sensitivity as well as specificity for the diagnosis of schizophrenia in the acute phase, such as studies of incidence. It also suggests that dropping the 6 month duration criterion should be considered for a future " $D S M-V$."

Tony Kendrick, MD St. George's Hospital Medical School London, UK

1 Crichton P. First-rank symptoms or rank and-file symptoms? Br J Psychiatry 1996;169:537-40. symptoms? Br Psychiatry 1996;169:537-40.
American Psychiatric Association. Diagnostic and Statistical Manual of Mental Disorders (4th edition). Washington, DC: American Psychiatric Association, 1994. 